\title{
CLONAGE DE L'IGF-I ET DE SON RÉCEPTEUR CHEZ LE TURBOT (PSETTA MAXIMA).
}

\author{
G. ELIES (1), H. DUVAL (1), L. GROIGNO (1), J. WOLFF (2), G. BOEUF (3) \\ et D. BOUJARD (1)
}

(1) UPRES-A 6026 CNRS / Université de Rennes 1, Biologie Cellulaire et Reproduction, Equipe Canaux et Récepteurs Membranaires, Campus de Beaulieu, 35042 Rennes Cedex, France.

(2) Université de Bretagne Sud, Centre Universitaire Scientifique de Vannes, Laboratoire de Biologie Moléculaire, 1 rue de la Loi, 56000 Vannes, France.

(3) IFREMER, Station Ressources Vivantes, B.P. 70, 29280 Plouzane, France.

\section{RÉSUMÉ}

Les IGF, leurs récepteurs et leurs protéines de liaison constituent une famille moléculaire qui joue un rôle essentiel dans la régulation de la croissance et du développement. Nous nous sommes intéressés à la caractérisation moléculaire de l'IGF-I et de son récepteur (IGF-1R) chez le turbot (Psetta maxima), un poisson plat téléostéen. Par RT-PCR, nous avons cloné un ADNc codant pour les quatre domaines de I'IGF-I mature. La protéine présente 70 à $96 \%$ d'identité avec les autres IGF-I de vertébrés. Le clonage de la séquence codante entière du récepteur a nécessité la construction d'une banque d'ADNc d'embryons de turbots. Le récepteur est organisé en domaines dont la taille et les caractéristiques sont semblables à celles des autres récepteurs aux IGF de type 1 décrits chez les mammifères et le poulet. La région catalytique est particulièrement conservée. Dans certains domaines, notamment dans la région C-terminale du précurseur, quelques différences qui pourraient avoir une importance fonctionnelle sont cependant observées. Enfin, par RT-PCR, nous avons mis en évidence une régulation ontogénique du statut de polyadénylation des ARNm IGF-1R.

\section{TURBOT (PSETTA MAXIMA) IGF-I AND RECEPTOR cDNAS CLONING.}

\section{ABSTRACT}

The IGFs, their receptors and their binding proteins constitute a family of cellular modulators that play essential functions in the regulation of growth and development. In our study, we have carried out the molecular characterization of the IGF-I and its receptor (IGF-1R) from the turbot (Psetta maxima), a teleost flatfish.

By RT-PCR, we have cloned an IGF-I CDNA. The deduced peptide shows $70-96 \%$ identity with the other vertebrate IGF-I sequences previously described. We have also cloned a cDNA encoding the IGF type 1 receptor precursor from an embryos cDNA library. The deduced IGF-1R primary sequence contains all the topological features characteristic 
of the IGF-1R. Indeed, the turbot receptor is highly conserved compared to its mammalian counterparts, particularly within the catalytic domain. Some differences were nevertheless observed in the primary sequence that may affect the function of the receptor. Finally, by using an RT-PCR approach, we have shown that IGF-1R mRNA polyadenylation status varies according to the developmental stage.

\section{INTRODUCTION}

Chez l'ensemble des vertébrés, la régulation de la croissance est sous le contrôle de l'axe somatotrope, mais aussi de l'axe thyréotrope et dans une moindre mesure de l'axe gonadotrope. L'hormone de croissance $(\mathrm{GH})$ joue un rôle central dans ce phénomène. Elle exerce néanmoins la majorité de ces effets somatogéniques de façon indirecte, via la stimulation de la production hépatique d'IGF-I ("Insulin-like Growth Factor-1 "). C'est ce dernier qui assure alors le contrôle de la prolifération et de la différenciation cellulaires (revue par HUMBEL, 1990). L'IGF-I, tout comme I'IGF-II, sont aussi sécrétés par de nombreux autres types cellulaires et exercent ainsi des actions paracrines et autocrines complémentaires de leur rôle endocrine joué au sein de l'axe somatotrope (HOLLY et WASS, 1989).

Les IGF sont l'une des composantes d'un système complexe incluant également des protéines de liaison, les IGF-BP ("IGF-Binding Proteins "), et des récepteurs membranaires spécifiques de haute affinité.

Chez les mammifères, sept IGF-BP distinctes ont été caractérisées (revue par JONES et CLEMMONS, 1995 ; OH et al., 1996). Outre leur rôle dans le transport des IGF, ces molécules sont capables de coordonner et de réguler l'interaction des IGF avec leurs récepteurs. Chez les poissons, l'existence de protéines de liaison a également été démontrée par des études de liaison d'hormones hétérologues marquées. Selon les espèces, un nombre variable d'IGF-BP a été caractérisé (KELLEY et al., 1992 ; ANDERSON et al., 1993), sans que leurs séquences ni leurs rôles éventuels dans la modulation des effets biologiques des IGF ne soient connus.

On possède en revanche plus de données sur les IGF. II existe, comme chez les autres vertébrés, deux IGF dont les ADNc ont été clonés chez plusieurs espèces de téléostéens (IGF-I : CAO et al., 1989 ; DUGUAY et al., 1992, 1996 ; SHAMBLOTT et CHEN, 1992 ; WALLIS et DEVLIN, 1993 ; MCRORY et SHERWOOD, 1994 ; REINECKE et al., 1997 ; IGF-II : SHAMBLOTT et CHEN, 1992 ; GENTIL et al., 1996 ; DUGUAY et al., 1996 ; CHEN et al., 1997). MORIYAMA et al. (1994) et GENTIL et al. (1996) ont mis au point des dosages radio-immunologiques (respectivement pour l'IGF-I et pour l'IGF-II) qui ont été utilisés pour la mesure des taux plasmatiques des IGF ; mais certains problèmes méthodologiques d'extraction des IGF-BP n'étaient pas toujours résolus, aussi l'interprétation physiologique ne s'est pas toujours avérée aisée. D'autres études, réalisées par Northern-blot ou par "RNase protection assay ", ont permis de mettre en évidence, dans certaines conditions telles que le jeûne ou la smoltification, des modifications des taux d'ARN messagers dans le foie (DUAN et PLISETSKAYA, 1993 ; DUAN et al., 1995), dans les branchies (SAKAMOTO et al., 1995) et dans les cellules testiculaires (LE GAC et al., 1996).

La plupart des effets cellulaires de l'IGF-I, mais aussi de l'IGF-II sont transmis, chez les mammifères, par l'intermédiaire du récepteur aux IGF de type 1 (IGF-1R). Chez ces derniers, I'IGF-II se lie également avec une forte affinité sur un récepteur aux IGF de type 2, qui est identique au récepteur au Mannose-6-Phosphate (M-6-P/IGF-2R). Cependant, cette interaction permettrait uniquement une régulation du taux de peptide 
libre. De plus, le récepteur au mannose-6-phosphate est incapable de lier l'IGF-ll chez le poulet (CANFIELD et KORNFELD, 1989), le xénope (KIESS et al., 1994), et très vraisemblablement chez les poissons (DRAKENBERG et al., 1993 ; LE GAC et al., 1996). Le récepteur aux IGF de type 1, qui appartient à la famille des tyrosines kinases, est une protéine glycosylée d'environ $300 \mathrm{kDa}$. Initialement synthétisé sous la forme d'un précurseur constitué d'une seule chaîne polypeptidique, il est ensuite clivé au niveau d'un site protéolytique localisé à la jonction des sous-unités $\alpha$ et $\beta$. Le récepteur fonctionnel est un hétérotétramère constitué de deux sous-unités $\alpha$ extracellulaires et de deux sous-unités $\beta$ transmembranaires, reliées par des ponts disulfures (revue par LEROITH et al., 1995). La partie $\alpha$ est responsable de la fixation des ligands tandis que la partie $\beta$ possède un domaine catalytique générant une activité tyrosine kinase. Chez les poissons osseux, les données concernant IGF-1R, très limitées, sont le fruit d'expériences de liaison de ligands, homologues ou hétérologues, sur des préparations membranaires brutes ou semi-purifiées. Des sites de liaison spécifiques de l'IGF-I ont ainsi été décrits dans le foie, les muscles squelettiques ou cardiaques, le cerveau et les gonades (DRAKENBERG et al., 1993 ; GUTIERREZ et al., 1993, 1995 ; LOIR et LE GAC, 1994 ; BLAISE et al., 1995 ; PARRIZAS et al., 1995 ; LE GAC et al., 1996 ; LEIBUSH et al., 1996).

Bien que les molécules impliquées dans la régulation de la croissance soient très semblables chez l'ensemble des vertébrés, les poissons présentent certaines particularités qui font qu'il est difficile d'extrapoler les résultats obtenus chez les mammifères. En particulier, le processus de croissance des poissons apparaît continu et surtout lié à des phénomènes d'hyperplasie cellulaire. Du fait de la complexité du système IGF (actions endocrine, paracrine et autocrine, interactions multiples entre les IGF et les IGF-BP), nous avons choisi de focaliser notre attention sur le récepteur, qui occupe une position clé dans ce système, et de développer les outils moléculaires homologues afin de pouvoir aborder, à terme, l'étude de IGF-1R et de ses ligands au cours de la croissance chez le turbot (Psetta maxima). Nous présentons ici les premiers résultats obtenus après clonage des ADNc correspondant à l'IGF-I et à son récepteur.

\section{MATÉRIEL ET MÉTHODES}

\section{Animaux et traitements}

Cette étude a été menée sur des embryons, des larves et des juvéniles de turbot d'environ $100 \mathrm{~g}$, gracieusement mis à notre disposition par la station IFREMER de Brest. Les juvéniles sont préacclimatés à leur structure d'élevage définitive (bassins de $1 \mathrm{~m}^{2}$, photopériode naturelle, salinité $34 \%$, température $17^{\circ} \mathrm{C}$ ) environ 3 semaines avant le début des expérimentations. Quelques poissons ont ensuite subi deux injections de $\mathrm{GH}$ $(0,5 \mu \mathrm{g} / \mathrm{kg})$ par semaine, puis ont été sacrifiés $24 \mathrm{~h}$ après la $9^{\mathrm{e}}$ injection.

\section{Amorces}

Trois couples d'amorces (Eurogentec) ont été utilisés. Pour l'amplification de l'ADNc IGF-I de turbot, les amorces IGF-ID et IGF-IE ont été choisies à partir de la séquence de I'IGF-I de truite arc-en-ciel. Elles permettent l'amplification de toute la séquence du peptide mature. L'amplification de la quasi-totalité du domaine tyrosine kinase de IGF-1R (sonde 1, Figure 3) a été réalisée à l'aide des amorces dégénérées 015 et Ol6. Cette séquence a ensuite été étendue en 5' grâce à une amorce spécifique antisens (O/10) et à une amorce dégénérée sens (OIC2). Tous ces oligonucléotides possèdent à leur extrémité 5 ' une séquence spécifique de 12 bases contenant des résidus dUMP qui, lorsqu'ils sont traités à l'« Uracyl DNA glycosylase ", deviennent abasiques et permettent l'insertion orientée des fragments amplifiés dans un vecteur pAMP1. 


\begin{tabular}{|cccc|}
\hline amorce & séquence $\left(5^{\prime} \cdot 3^{\prime}\right)$ & S ou AS & $\operatorname{Tm}\left({ }^{\circ} \mathbf{C}\right)$ \\
\hline IGF-ID & CUA CUA CUA CUA TCC TAA CCC TGA CTT CGG CG & S & 59 \\
IGF-IE & CAU CAU CAU CAU TTC TTG GCA TGT CTG TGT GGC & AS & 58 \\
\hline OI5 & CUA CUA CUA CUA TCT GTS AAC CCN GAR TAY TTY & S & 58 \\
OI6 & CAU CAU CAU CAU AGG CCK CCY TTC ATV ACR AA & AS & 59 \\
\hline OI10 & CAU CAU CAU CAU TCT TTG ACC ACG CCC TTC GC & AS & 66 \\
OIC2 & CUA CUA CUA CUA ACA GAR TAY GAY GGN CAR GA & S & 58 \\
\hline
\end{tabular}

Abréviations pour les amorces dégénérées : $R=[A, G] ; Y=[C, T] ; K=[T, G] ; S=[C, G]$; $V=[A, C, G] ; N=[A, C, G, T] . S$ ou $A S$ : amorce Sens ou AntiSens.

\section{Synthèse d'ADNc par RT-PCR}

L'extraction des ARN totaux a été réalisée selon la technique décrite par AUFFRAY et ROUGEON (1980). Les réactions de transcription inverse (RT) sont réalisées à partir de $10 \mu \mathrm{g}$ d'ARN total en présence de $50 \mathrm{ng}$ d'hexamères aléatoires ou $0,5 \mu \mathrm{M}$ d'oligo(dT), $0,5 \mathrm{mM}$ de chaque dNTP, $4 \mu \mathrm{l}$ de tampon et 200 unités de transcriptase inverse "Superscript" RNase $H^{-}$" (Gibco BRL) dans un volume réactionnel de $20 \mu \mathrm{l}$. La polymérisation est effectuée à $37^{\circ} \mathrm{C}$ durant 1 heure.

Le milieu de PCR $(50 \mu \mathrm{l})$ se compose de $10 \mu \mathrm{l}$ de milieu de RT, de 35 pmoles de chaque amorce (sauf pour les amorces O15 et Ol6: 175 pmoles), de $5 \mu$ de tampon de réaction, de 1,5 $\mathrm{mM}$ de $\mathrm{MgCl}_{2}$, de $150 \mu \mathrm{M}$ de chaque dNTP et de 2,5 unités de "Goldstar DNA Polymerase " (Eurogentec). La réaction de polymérisation est caractérisée par la succession de 30 (pour IGF-1R) ou de 45 cycles (pour IGF-I) constitués chacun des étapes suivantes : dénaturation $\left(94^{\circ} \mathrm{C}, 1 \mathrm{~min} 30\right)$; hybridation $\left(50\right.$ ou $\left.55^{\circ} \mathrm{C}, 1 \mathrm{~min} 30\right)$; élongation $\left(72^{\circ} \mathrm{C}, 1 \mathrm{~min} 30\right)$.

\section{Clonage et séquençage}

Les produits purifiés de PCR ont été clonés dans le vecteur pAMP1 ("Clone AMP System ", Gibco BRL), suivant le protocole recommandé par le fournisseur. Après leur transformation par électroporation, les bactéries recombinantes sont sélectionnées, puis l'ADN plasmidique est purifié par les techniques classiques et séquencé selon la méthode de SANGER et al. (1977) à l'aide du kit « Sequenase II » (USB).

\section{Construction et criblage d'une banque d'ADNc d'embryons de turbots}

La synthèse des $A D N c$ est réalisée à partir de $2 \mu \mathrm{g}$ d'ARN poly $(A)+$ d'embryons de turbots âgés de 48 heures. Cette synthèse ainsi que l'insertion des ADNc dans le phage $\lambda$ gt 10 sont réalisées à l'aide du kit "Riboclone ADNc Synthesis " (Promega). Après encapsidation (" Gigapack II Gold Packaging Extract », Stratagène), les phages ont servi à infecter des bactéries $\mathrm{C}_{600} \mathrm{Hfl}$. La banque a été étalée en totalité, sans être amplifiée (titre : $1,5.10^{5} \mathrm{pfu} / \mathrm{\mu g}$ de vecteur).

Les deux sondes IGF-1R amplifiées par RT-PCR ont successivement été utilisées pour cribler la banque. Elles ont été marquées au dCTP $\left[\alpha^{32} \mathrm{P}\right]$ par la technique d'amorçage au hasard. Après préhybridation, les filtres ont été hybridés pendant $16 \mathrm{~h}$ à $65^{\circ} \mathrm{C}$ dans un milieu 6 X SSPE, $5 \times$ Denhardt's, 0,5\% SDS, $100 \mu \mathrm{g} / \mathrm{ml}$ d'ARNt de levure, contenant la sonde dénaturée à raison de $10^{6} \mathrm{cpm} / \mathrm{ml}$ de milieu. Des lavages stringents ont ensuite été réalisés, puis les filtres ont été exposés contre un film Kodak XAR- 5 à $-70^{\circ} \mathrm{C}$ pendant $24 \mathrm{~h}$. 


\section{RÉSULTATS - DISCUSSION}

\section{Clonage et séquençage d'un ADNc correspondant à l'IGF-1 de turbot}

A partir d'ARN totaux de foie de turbots traités à la $\mathrm{GH}$, nous avons pu, par RT-PCR, amplifier un ADNc d'environ $300 \mathrm{pb}$. La séquence peptidique, déduite après séquençage de ce clone, comprend la totalité de l'IGF-I mature. Constitué de 68 acides aminés, il présente un pourcentage de similarité compris entre 70 et $96 \%$ avec les autres séquences connues de vertébrés. Comme chez les autres espèces, ce peptide peut être divisé en quatre domaines. Les domaines $A$ et $B$ sont les plus conservés avec respectivement 86 et $90 \%$ d'identité avec la séquence humaine (Tableau I). Chez les mammifères, ces deux régions jouent un rôle important dans la liaison de l'IGF-I avec son récepteur et avec les protéines de liaison (CASCIERI et BAYNE, 1994).

\section{Tableau I}

Pourcentages d'identité entre l'IGF-I de turbot et celui d'autres vertébrés.

Table I

Percent IGF-I sequence identity between turbot and other vertebrate species.

\begin{tabular}{|c|c|c|c|c|}
\hline Domaines & B & C & A & D \\
\hline Dorade & 93,1 & 100 & 95,2 & 100 \\
\hline Tilapia & 96,6 & 91,6 & 95,2 & 50 \\
\hline Truite & 100 & 66,7 & 95,2 & 62,5 \\
\hline Saumon & 100 & 66,7 & 95,2 & 62,5 \\
\hline Xénope & 96,6 & 66,7 & 85,7 & 62,5 \\
\hline Poulet & 93,1 & 41,7 & 90,5 & 50 \\
\hline Homme & 89,7 & 41,7 & 85,7 & 50 \\
\hline
\end{tabular}

Tous les acides aminés décrits comme étant déterminants pour la conformation ou pour l'activité biologique de la molécule sont présents dans la séquence du turbot (Figure 1). On retrouve en effet les six résidus cystéines intervenant dans la formation des ponts disulfures intra- ou inter-domaines, les acides aminés leucines, valines et isoleucines qui contribuent à la formation du noyau hydrophobe de I'IGF-I, ainsi que les résidus Phe23, Tyr24 et Phe25 qui sont impliqués dans la liaison du peptide à son récepteur. L'absence de deux acides aminés dans le domaine $C$ par rapport aux séquences mammaliennes est également observée chez le tilapia et la dorade, mais n'existe ni chez le saumon, ni chez la truite.

\section{Caractérisation de l'ARN messager du récepteur aux IGF de type 1}

Chez les mammifères, l'ARNm IGF-1R se caractérise notamment par sa taille importante $(11 \mathrm{~kb})$ mais aussi par sa distribution, faible quantitativement mais néanmoins ubiquitaire (revue par LEROITH et al., 1995). Notre première démarche a consisté à amplifier un ADNc nous permettant d'obtenir des informations sur la nature de cet ARN messager chez le turbot. Par RT-PCR, à l'aide des amorces dégénérées OI5 et O16, nous avons obtenu un fragment d'ADNc de $790 \mathrm{pb}$ couvrant la quasi-totalité du domaine tyrosine kinase (sonde 1, Figure 3). En utilisant cette sonde, nous avons réalisé des 
homme

poulet

xénope

saumon

tilapia

dorade

turbot
DOMAINE B

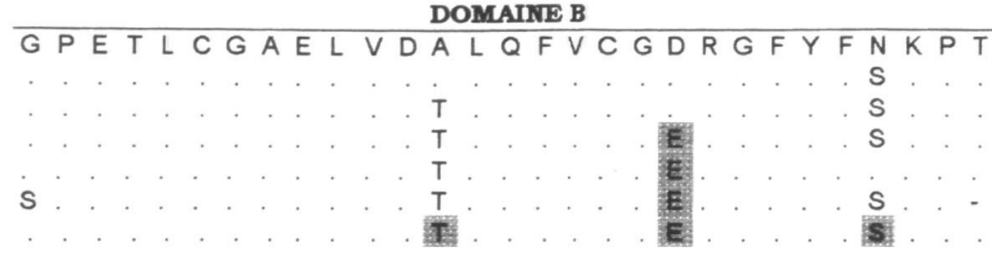

G Y G S S S R

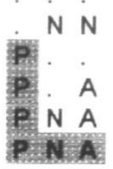

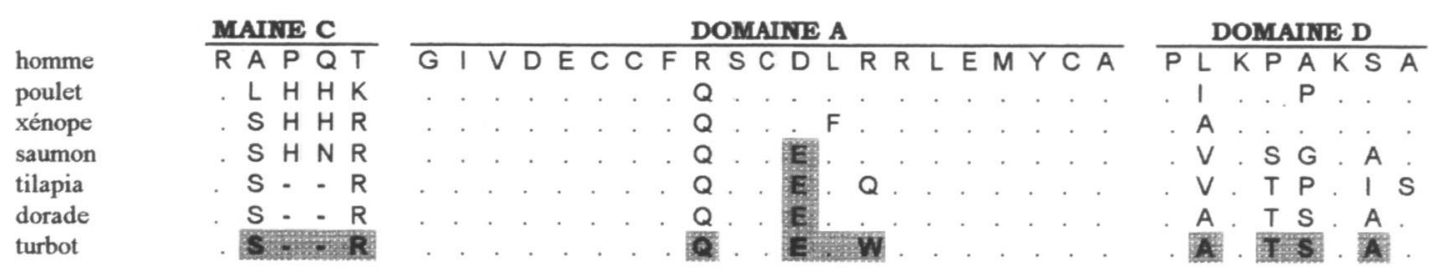

\section{Figure 1}

Alignement et comparaison des séquences protéiques de différents IGF-I. homme (RINDERKNECHT et HUMBEL, 1978) - poulet (KAJIMOTO et ROTWEIN, 1989) - xénope (KAJIMOTO et ROTWEIN, 1990) - saumon (CAO et al., 1989) truite (SHAMBLOTT et CHEN, 1992) - tilapia (REINECKE et al., 1997) - dorade (DUGUAY et al., 1996).

\section{Figure 1}

\section{Alignment and comparison of different IGF-I primary sequences.} human (RINDERKNECHT and HUMBEL, 1978) - chicken (KAJIMOTO and ROTWEIN, 1989) - xenopus (KAJIMOTO and ROTWEIN, 1990) - salmon (CAO et al., 1989) tilapia (REINECKE et al., 1997) - gilthead seabream (DUGUAY et al.,1996).

expériences de Northern-blot et ainsi visualisé un messager unique de $13 \mathrm{~kb}$, plus fortement exprimé dans la branchie que dans le muscle, et à peine détectable dans le foie (ELIES et al., 1996). La grande taille de l'ARNm que nous avons détecté suggère que celui-ci possède, comme chez les mammifères, une très longue région 3 ' non traduite (3'NTR).

Nous avions montré au laboratoire que le statut de polyadénylation des ARNm IGF-1R semblait être régulé différemment selon le stade de développement chez le xénope (GROIGNO et al., 1996). Nous avons donc entrepris une étude du statut de polyadénylation des ARNm IGF-1R de turbots au cours des développements embryonnaire et larvaire (stade 4 cellules, gastrula, larves de 10 jours et en fin de métamorphose) ainsi que dans des ovocytes II et dans plusieurs tissus somatiques adultes (muscle, foie et encéphale), afin de déterminer les stades du développement où le messager est présent sous une forme polyadénylée. Pour chacune des préparations d'ARN totaux, une réaction de transcription inverse a été effectuée à l'aide soit d'oligo(dT), soit d'hexamères aléatoires. A l'issue de ces deux réactions de transcription inverse, une PCR a été réalisée à l'aide d'oligonucléotides spécifiques. Les résultats obtenus sont présentés à la Figure 2. Avec les hexamères, un fragment est amplifié à tous les stades embryonnaires ou larvaires et dans tous les tissus adultes testés. Avec les oligo(dT), seuls les milieux issus d'ovocytes II et des deux stades embryonnaires permettent l'amplification du fragment attendu (ELIES et al., 1996). Dans les tissus somatiques adultes et dans les deux stades larvaires, l'ARNm IGF-1R n'est pas amplifié. L'état de polyadénylation de ce messager n'est donc pas constant : polyadénylé dans les ovocytes et les premiers stades embryonnaires, le messager apparaît comme déadénylé chez les larves et dans les tissus somatiques des turbots adultes. 


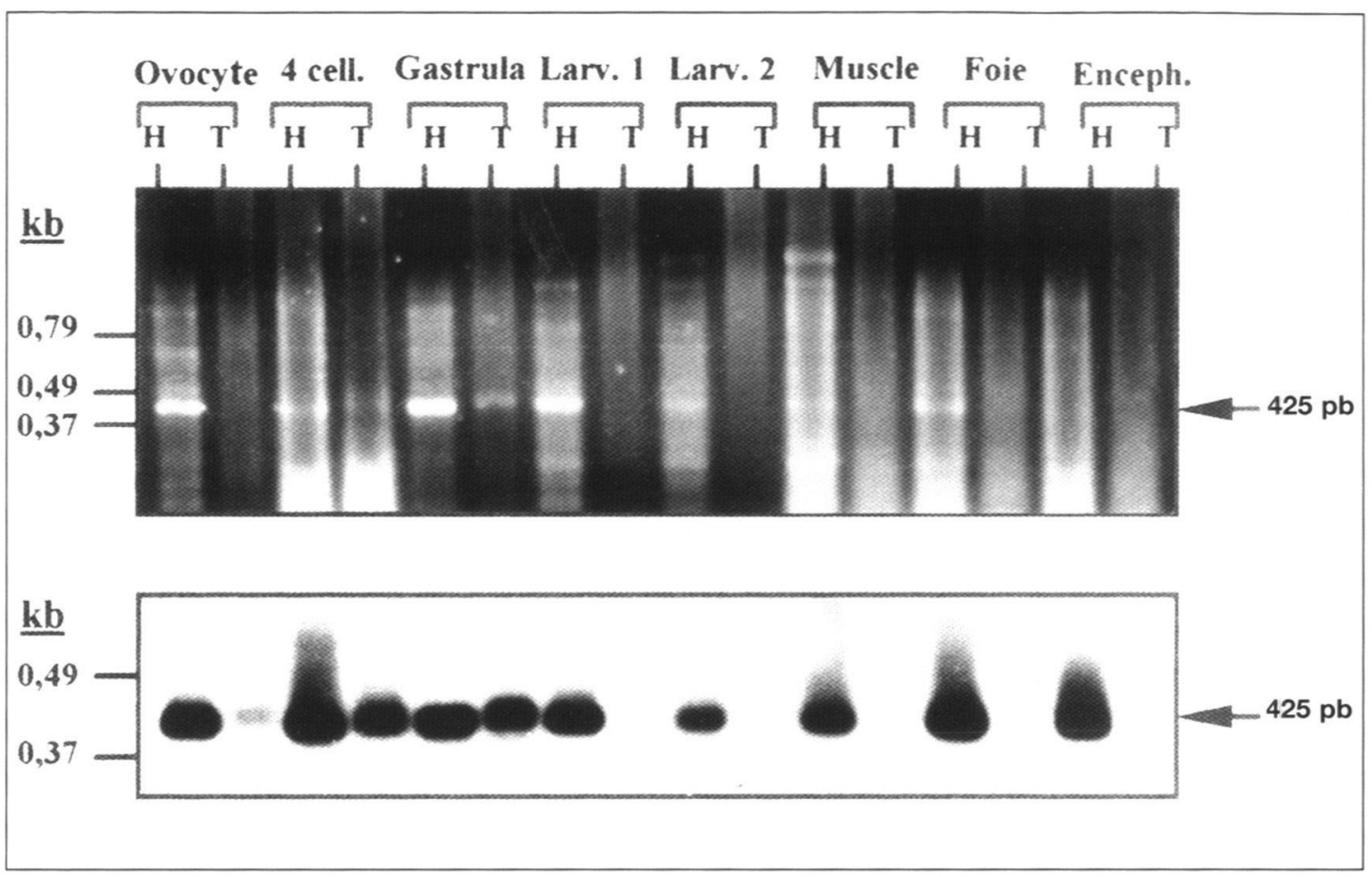

Figure 2

Etat de polyadénylation des ARNm IGF-1R au cours du développement.

La réaction de transcription inverse est réalisée sur $10 \mu \mathrm{g}$ d'ARN totaux d'ovocytes II, d'embryons au stade 4 cellules ou gastrula, de larves de 10 jours ou en fin de métamorphose, de muscle, de foie ou d'encéphale, en utilisant soit des hexamères aléatoires (H), soit des amorces oligo(dT) (T). Après 35 cycles de PCR effectués avec un couple d'amorces spécifiques de l'ADNc IGF-1R, les produits d'amplification ont été analysés par électrophorèse dans un gel d'agarose à 1,5\% (photo du haut). Après transfert des ADNc sur une membrane de nylon, ceux-ci ont été hybridés avec une sonde IGF-1R de turbot afin d'augmenter la sensibilité de détection. (D'après ELIES et al., 1996).

Figure 2

Polyadenylation status of IGF-1R mRNAs during development.

cDNAs were synthesized from $10 \mu \mathrm{g}$ total RNA from oocytes II, embryos at four cells or gastrula stages, 10-day-old or end metamorphosis larvae, muscle, liver and encephalon, with either random hexamers $(H)$ or oligo(dT) primers (T). After 35 PCR cycles using a specific set of primers, amplified products were analysed by electrophoresis on a $1.5 \%$ agarose gel (upper panel). After blotting on a nylon membrane, the cDNAs were hybridized with a turbot IGF-1R probe to increase the detection sensitivity. (From ELIES et al., 1996). type 1

3. Clonage et séquençage d'un ADNc correspondant au récepteur aux IGF de

La banque d'ADNc a été réalisée à partir d'ARN messagers prélevés sur des embryons âgés de $48 \mathrm{~h}$, en utilisant des hexamères aléatoires pour s'affranchir de la longueur de la région 3' NTR et pouvoir cloner la séquence codante entière $(4,1 \mathrm{~kb})$. Construite dans le phage $\lambda . g t 10$, elle présente un titre de $1,5.10^{5} \mathrm{pfu} / \mathrm{\mu g}$ de vecteur. Elle a été entièrement étalée, sans amplification préalable, de façon à éviter la perte des 
messagers rares. Le criblage de cette banque avec la sonde obtenue par RT-PCR (sonde 1) a abouti à l'obtention de sept clones correspondant à de l'IGF-1R, ces clones couvrant environ $0,2 \mathrm{~kb}$ de sous-unité $\alpha$, toute la sous-unité $\beta$ (soit $2 \mathrm{~kb}$ ) et $1,5 \mathrm{~kb}$ de séquence 3' NTR (Figure 3). Une deuxième sonde, localisée dans la partie $\alpha$ du récepteur, a ensuite été produite (par RT-PCR en utilisant les amorces OIC2 et Ol10, suivie du clivage du fragment amplifié par l'enzyme BamHI) et utilisée pour un deuxième criblage de la banque. Deux clones, codants pour la totalité de la partie $\alpha$ et pour environ $1 \mathrm{~kb}$ de la partie 5' non traduite, ont été isolés puis séquencés. L'ensemble des clones obtenus couvre donc la séquence codante entière de l'ADNc du récepteur aux IGF de type 1, ainsi que deux portions d'environ $1 \mathrm{~kb}$ des régions 5 ' et 3 ' NTR. Cette séquence est publiée dans la banque de données EMBL sous le numéro d'accession AJ224993. La séquence peptidique a été déduite et les pourcentages d'identité avec le récepteur humain sont présentés, région par région, à la Figure 4.

La sous-unité $\alpha$ du récepteur possède un domaine riche en cystéines qui présente un pourcentage global d'identité assez modéré avec les séquences des récepteurs mammaliens, malgré la localisation strictement identique des 24 cystéines qui sont répertoriées dans ce domaine. La comparaison des différentes séquences connues ne permet pas de définir plus précisément le site de liaison de l'IGF-I, mais la parfaite conservation de ces cystéines indique qu'elles doivent jouer un rôle important dans le maintien d'une structure tridimensionnelle indispensable à la fixation du ligand.

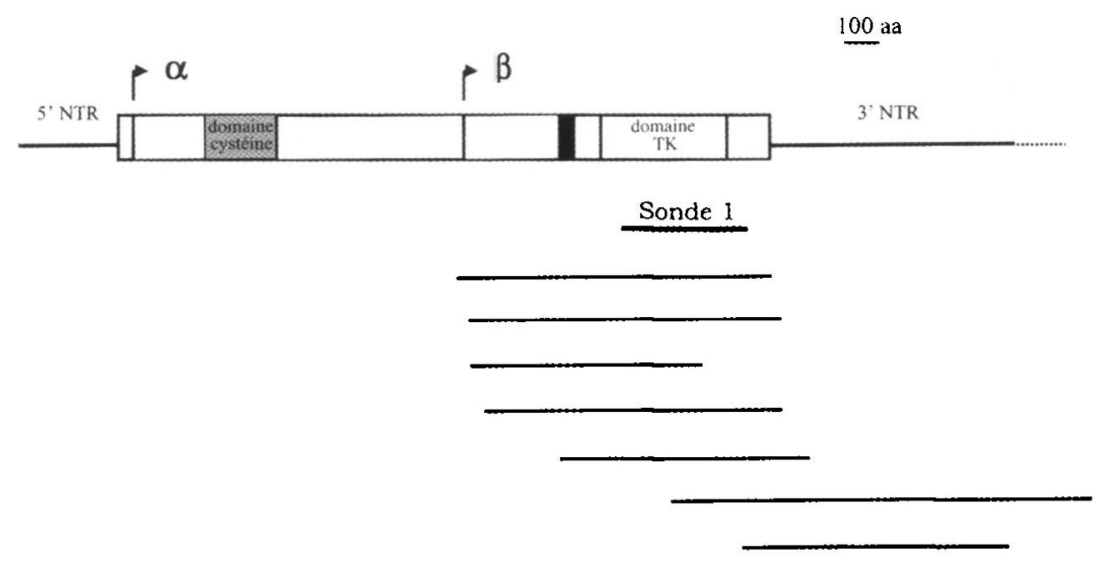

Sonde 2

Figure 3

Localisation des clones IGF-1R isolés après le criblage de la banque d'ADNc d'embryons.

Un schéma de la structure de l'ADNc IGF-1R humain est présenté. Les régions traduites sont représentées par un rectangle. La position des deux amorces amplifiées par PCR est également indiquée. Les clones chevauchants obtenus par le criblage de la banque d'ADNc d'embryons sont positionnés par des traits horizontaux.

\section{Figure 3}

IGF-1R cDNA clones isolated from the screening of the CDNA embryos library. Schematic diagram of the CDNA structure of human IGF-1R is shown. Translated sequences are boxed. The position of the two PCR-amplified probes is indicated. Overlapping cDNA clones obtained by screening of the embryos cDNA library are shown below. 
Le domaine catalytique est très conservé. Le récepteur du turbot possède dans ce domaine tous les résidus invariants décrits chez l'ensemble des protéines kinases (HANKS et al., 1988). Trois tyrosines sont en particulier trouvées en position identique à celles correspondant au site majeur d'autophosphorylation chez les mammifères. La plupart des autres tyrosines dénombrées dans ce domaine chez l'homme sont déjà présentes dans la séquence du turbot. La conservation de ces résidus, pour lesquels aucune expérience de mutagenèse ne semble avoir été réalisée, laisse toutefois présumer qu'ils pourraient exercer une fonction importante. La région juxtamembranaire est une zone également bien conservée. Les motifs NPXY et GXLY sont présents et pourraient ainsi jouer un rôle dans l'endocytose du récepteur. La tyrosine du motif NPXY pourrait également, comme chez les mammifères, être impliquée dans la phosphorylation de I'IRS-1, l'un des tous premiers substrats du récepteur. La partie extracellulaire de la sous-unité $\beta$ est en revanche assez différente chez le turbot. On retrouve néanmoins plusieurs sites potentiels de $\mathrm{N}$-glycosylation en position quasi identique à ceux observés chez les mammifères. Enfin, la région C-terminale possède une insertion de 43 acides aminés qui n'est observée dans aucune autre séquence de vertébrés. La présence de longues insertions $\mathrm{C}$-terminales a en revanche également été décrite dans les deux séquences de récepteurs "insulin-like " d'invertébrés connues à ce jour (drosophile et limnée). Contrairement à ce qui est observé dans le cas du récepteur de turbot, leurs insertions se caractérisent par l'existence de plusieurs tyrosines dont la fonction exacte demeure toutefois imprécise.

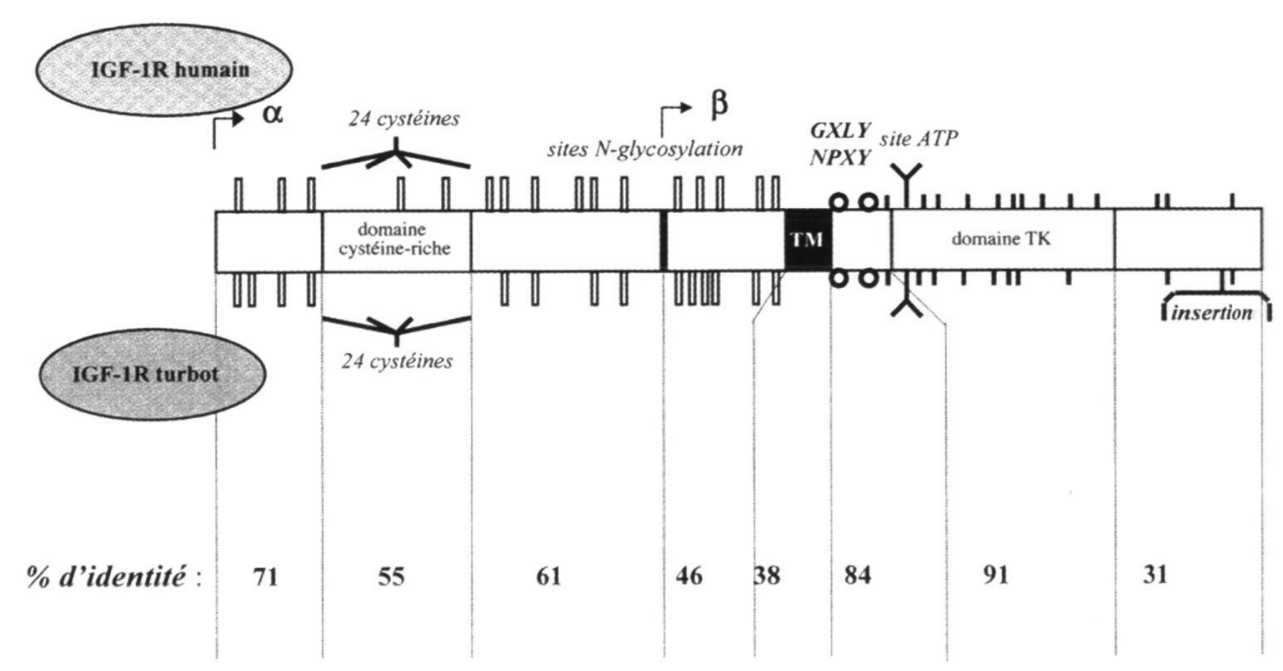

\section{Figure 4}

Comparaison des préprorécepteurs aux IGF de type 1 de l'homme et du turbot. Les sites potentiels de $\mathrm{N}$-glycosylation sont indiqués par des barres verticales vides, les tyrosines intracellulaires par des traits noirs épais, les motifs juxtamembranaires (GXLY et NPXY) par des ronds et enfin, les résidus impliqués dans la fixation de l'ATP par un trait fourchu. TM : domaine transmembranaire ; TK : domaine tyrosine kinase.

\section{Figure 4}

Comparison between human and turbot IGF-1R precursors.

Potential $\mathrm{N}$-glycosylation sites are indicated by open dashes, intracellular tyrosines by black bars, the juxtamembrane motifs (GXLY et NPXY) by open circles and finally, the residues involved in ATP binding by branching dash. TM : transmembrane domain ; TK : tyrosine kinase domain. 


\section{CONCLUSION}

L'obtention de la première séquence codante complète du récepteur aux IGF de type 1 chez un poisson démontre que le récepteur, comme le ligand, ont subi peu de modifications au cours de l'évolution, ce qui témoigne de leur importance biologique. Comme chez presque toutes les autres espèces (à l'exception de l'homme, chez qui un deuxième messager peut être observé), l'ARN messager du récepteur est unique. La longueur importante de cet ARNm, qui s'explique par l'existence de régions 3 ' et 5 ' non traduites de grande taille, est donc une caractéristique commune à tous les vertébrés. Des études plus détaillées seront nécessaires pour déterminer l'importance de ces régions dans la régulation de l'expression du gène du récepteur aux IGF de type 1. D'ores et déjà, la mise en évidence d'une modification de l'ARNm au cours des premières étapes du développement semble indiquer que ce phénomène pourrait jouer un rôle primordial dans le contrôle de l'expression de ce gène. Etant donné l'importance des régulations paracrine et autocrine dans le fonctionnement du système IGF, il est fondamental de pouvoir quantifier les ARN messagers. L'obtention de sondes homologues rend ce travail possible. De même, la possession de ligands recombinants homologues (IGF-l et IGF-II) permettra également de réaliser des expériences de liaison sur différents tissus, afin de pouvoir corréler la présence de l'ARNm IGF-1R à celle de la protéine.

\section{REMERCIEMENTS}

Ce travail a été soutenu financièrement par l'IFREMER (contrat 96.2.527159) et la fondation Langlois. Nous remercions également L. Communier pour les photographies.

\section{BIBLIOGRAPHIE}

ANDERSON T.A., BENNETT L.R., CONLON M.A., OWENS P.C., 1993. Immunoreactive and receptor-active insulin-like growth factor-I (IGF-I) and IGF-binding protein in blood plasma from the freshwater fish Macquaria ambigua (golden perch). J. Endocrinol., 136, 191-198.

AUFFRAY C., ROUGEON F., 1980. Purification of mouse immunoglobulin heavy-chain messenger RNAs from total myeloma tumor RNA. Eur. J. Biochem., 107, 303-314.

BLAISE O., WEIL C., LE BAIL P.Y., 1995. Role of IGF-I in the control of GH secretion in rainbow trout (Oncorhynchus mykiss). Growth Regul., 5, 142-150.

CANFIELD W.M., KORNFELD S., 1989. The chicken liver cation-independent mannose-6-phosphate receptor lacks the high affinity binding site for insulin-like growth factor-II. J. Biol. Chem., 264, 7100-7103.

CAO Q.P., DUGUAY S.J., PLISETSKayA E., STEINER D.F., CHAN S.J., 1989. Nucleotide sequence and growth hormone-regulated expression of salmon insulin-like growth factor-I mRNA. Mol. Endocrinol., 3, 2005-2010.

CASCIERI M.A., BAYNE M.L., 1994. Analysis of the interaction of insulin-like growth factor-I (IGF-I) analogous with the IGF-I receptor and IGF-binding proteins. Horm. Res., 41, 80-86.

CHEN J.Y., CHANG C.Y., CHEN J.C., SHEN S.C., WU J.L., 1997. Production of biologically active recombinant tilapia insulin-like growth factor-ll polypeptides in Escherichia coli cells and characterization of the genomic structure of the coding region. DNA Cell Biol., 16, 883-892.

DRAKENBERG K., SARA V.P., FALKMER S., GAMMELTOFT S., MAAKE C., REINECKE M., 1993. Identification of IGF-1 receptors in primitive vertebrates. Regul. Pept., 43, 73-81. 
DUAN C., PLISETSKAYA E.M., 1993. Nutritional regulation of insulin-like growth factor-I mRNA expression in salmon tissues. J. Endocrinol., 139, 243-252.

DUAN C., PLISETSKAYA E.M., DICKHOFF W.W., 1995. Expression of insulin-like growth factor-I in normally and abnormally developing coho salmon (Oncorhynchus kisutch). Endocrinology, 136, 446-452.

DUGUAY S.J., PARK L.K., SAMADPOUR S., DICKHOFF W.W., 1992. Nucleotide sequence and tissue distribution of three insulin-like growth factor-I prohormones in salmon. Mol. Endocrinol., 6, 1202-1210.

DUGUAY S.J., LAI-ZHANG J., STEINER D.F., FUNKENSTEIN B., CHAN S.J., 1996. Developmental and tissue-regulated expression of IGF-I and IGF-II mRNAs in Sparus aurata. J. Mol. Endocrinol., 16, 123-132.

ELIES G., GROIGNO L., WOLFF J., BOEUF G., BOUJARD D., 1996. Characterization of the insulin-like growth factor type 1 receptor messenger in two teleost species. Mol. Cell. Endocrinol., 124, 131-140.

GENTIL V., MARTIN P., SMALL J., LE BAIL P.Y., 1996. Production of recombinant insulin-like growth factor-II in the development of a radioimmunoassay in rainbow trout (Oncorhynchus mykiss). Gen. Comp. Endocrinol., 104, 156-167.

GROIGNO L., BONNEC G., WOLFF J., JOLY J., BOUJARD D., 1996. Insulin-like growth factor-1 receptor (IGF-1R) messenger expression during oogenesis in Xenopus laevis. Endocrinology, 137, 3856-3863.

GUTIERREZ J., PARRIZAS M., CARNEIRO N., MAESTRO J.L., PLANAS J., 1993. Insulin and IGF-I receptors and tyrosine kinase activity in carp ovaries : changes with reproductive cycle. Fish Physiol. Biochem., 11, 247-254.

GUTIERREZ J., MAESTRO M.A., NAVARRO I., PLISETSKAYA E.M., 1995. Insulin and IGF-I binding and tyrosine kinase activity in fish heart. J. Endocrinol., 146, 35-44.

HANKS S.K., QUINN A.M., HUNTER T., 1988. The protein kinase family : conserved features and deduced phylogeny of the catalytic domains. Science, 241, 42-52.

HOLLY J.M.P., WASS J.A.H., 1989. Insulin-like growth factors : autocrine, paracrine or endocrine ? New perspectives of somatomedin hypothesis in the light of recent developments. J. Endocrinol., 122, 611-618.

HUMBEL R.E., 1990. Review : insulin-like growth factor-I and -II. Eur. J. Biochem., 190, 445-462.

JONES J.I., CLEMMONS D.R., 1995. Insulin-like growth factors and their binding proteins : biological actions. Endocr. Rev., 16, 3-34.

KAJIMOTO L., ROTWEIN P., 1989. Structure and expression of chicken insulin-like growth factor-I precursor. Mol. Endocrinol., 3, 1907-1913.

KAJIMOTO L., ROTWEIN P., 1990. Evolution of insulin-like growth factor-I (IGF-I) : structure and expression of an IGF-I precursor from Xenopus laevis. Mol. Endocrinol., 4, 217-226.

KELLEY K.M., GERHARD D.S., ARKINS S., LI Y.M., 1992. Growth hormone, prolactin, and insulin-like growth factors : new jobs for old players. Brain Behav. Immun., 6, 317-326.

KIESS W.L., YANG Y., KESSLER U., HOEFLICH A., 1994. Insulin-like growth factor-II (IGF-II) and the IGF-II/mannose-6-phosphate receptor : the myth continues. Horm. Res., 41, 66-73.

LE GAC F., LOIR M., LE BAIL P.Y., OLLITRAULT M., 1996. Insulin-like growth factor-I (IGF-I) mRNA and IGF-I receptor in trout testis and in isolated spermatogenic and sertoli cells. Mol. Reprod. Dev., 44, 23-35.

LEIBUSH B., PARRIZAS M., NAVARRO I., LAPPOVA Y., MAESTRO M.A., ENCINAS M., PLISETSKAYA E.M., GUTIERREZ J., 1996. Insulin and insulin-like growth factor-I 
receptors in fish brain. Regul. Pept., 61, 155-161.

LEROITH D., WERNER H., BEITNER-JOHNSON D., ROBERTS Jr. C.T., 1995. Molecular and cellular aspects of the insulin-like growth factor-l receptor. Endocr. Rev., 16, 143-163.

LOIR M., LE GAC F., 1994. Insulin-like growth factor-I and -II binding and action on DNA synthesis in rainbow trout spermatogonia and spermatocytes. Biol. Reprod., 51, 1154-1163.

McRORY J.E., SHERWOOD N.M., 1994. Catfish express two forms of insulin-like growth factor-l (IGF-I) in the brain. J. Biol. Chem., 269, 18588-18592.

MORIYAMA S., SWANSON P., NISHII M., TAKAHASHI A., KAWAUCHI H., DICKHOFF W.W., PLISETSKAYA E.M., 1994. Development of a homologous radioimmunoassay for coho salmon insulin-like growth factor-I. Gen. Comp. Endocrinol., 96, 149-161.

OH Y., NAGALLA S.R., YAMANADA Y., KIM H.S., WILSON E., ROSENFELD R.G., 1996. Synthesis and characterization of insulin-like growth factor-binding protein (IGFBP)7.recombinant human mac25 protein specifically binds IGF-I and -II. J. Biol. Chem., $271,30322-30325$.

PARRIZAS M., PLISETSKAYA E.M., PLANAS J., GUTIERREZ J., 1995. Abundant insulin-like growth factor-1 (IGF-1) receptor binding in fish skeletal muscle. Gen. Comp. Endocrinol., 98, 16-25.

REINECKE M., SCHMID A., ERMATINGER R., LOFFING-CUENI D., 1997. Insulin-like growth factor-I in the teleost Oreochromis mossambicus, the tilapia: gene sequence, tissue expression, and cellular localization. Endocrinology, 138, 36133619.

RINDERKNECHT E., HUMBEL R.E., 1978. The amino acid sequence of human insulin-like growth factor-I and its structural homology with proinsulin. J. Biol. Chem., 253, 2769-2776.

SAKAMOTO T., HIRANO T., MADSEN S.S., NISHIOKA R.S., BERN H.A., 1995. Insulin-like growth factor-I gene expression during parr-smolt transformation of coho salmon. Zool. Sci., 12, 249-252.

SANGER F., NICKLEN S., COULSON A.R., 1977. DNA sequencing with chain-terminating inhibitors. Proc. Natl. Acad. Sci. USA, 74, 5463-5467.

SHAMBLOTT M.J., CHEN T., 1992. Identification of a second insulin-like growth factor in a fish species. Proc. Natl. Acad. Sci. USA, 89, 8913-8917.

SHAMBLOTT M.J., CHENG C.M., BOLT D., CHEN T., 1995. Appearence of insulin-like growth factor mRNA in the liver and pyloric ceca of a teleost in response to exogenous growth hormone. Proc. Natl. Acad. Sci. USA, 92, 6943-6946.

WALLIS A.E., DEVLIN R.H., 1993. Duplicate insulin-like growth factor-I genes in salmon display alternative splicing pathways. Mol. Endocrinol., 7, 409-422. 